\title{
BMJ Open Protocol for a gallbladder cancer registry study in China: the Chinese Research Group of Gallbladder Cancer (CRGGC) study
}

\begin{abstract}
Tai Ren (D) , ${ }^{1,2}$ Yongsheng Li, ${ }^{2,3}$ Xi Zhang (D) , ${ }^{4}$ Yajun Geng, ${ }^{1,2}$ Ziyu Shao, ${ }^{1,2}$ Maolan Li, ${ }^{1,2}$ Xiangsong Wu, ${ }^{1,2}$ Xu-An Wang, ${ }^{2,3}$ Fatao Liu, ${ }^{2}$ Wenguang Wu, ${ }^{2,3}$ Yijun Shu, ${ }^{1,2}$ Runfa Bao, ${ }^{1,2}$ Wei Gong, ${ }^{1,2}$ Ping Dong, ${ }^{1,2}$ Xueyi Dang, ${ }^{5}$ Chang Liu, ${ }^{6}$ Changjun Liu, ${ }^{7}$ Bei Sun, ${ }^{8}$ Jun Liu, ${ }^{9}$ Lin Wang, ${ }^{10}$ Defei Hong, ${ }^{11}$ Renyi Qin (D),${ }^{12}$ Xiaoqing Jiang, ${ }^{13}$ Xuewen Zhang, ${ }^{14}$ Junmin Xu, ${ }^{15}$ Jianguang Jia, ${ }^{16}$ Bo Yang, ${ }^{17}$ Bing Li, ${ }^{18}$ Chaoliu Dai, ${ }^{19}$ Jingyu Cao, ${ }^{20}$ Hong Cao, ${ }^{21}$ Feng Tao, ${ }^{22}$ Zaiyang Zhang, ${ }^{23}$ Yi Wang, ${ }^{24}$ Huihan Jin, ${ }^{25}$ Hongyu Cai, ${ }^{26}$ Zhewei Fei, ${ }^{27}$ Jianfeng Gu, ${ }^{28}$ Wei Han, ${ }^{29}$ Xuedong Feng, ${ }^{30}$ Lu Fang, ${ }^{31}$ Linhui Zheng, ${ }^{32}$ Chunfu Zhu, ${ }^{33}$ Kunhua Wang, ${ }^{34}$ Xueli Zhang, ${ }^{35}$ Xiaoyong Li, ${ }^{36}$ Chong Jin, ${ }^{37}$ Yeben Qian, ${ }^{38}$ Yunfu Cui, ${ }^{39}$ Yuzhen Xu, ${ }^{40}$ Xiang Wang, ${ }^{41}$ Houbao Liu, ${ }^{42}$ Yawei Hua, ${ }^{43}$ Chao Liu, ${ }^{44}$ Jihui Hao, ${ }^{45}$ Chuanlei Wang, ${ }^{46}$ Qiyun Li, ${ }^{47}$ Xun Li ${ }^{48}$ Jiansheng Liu, ${ }^{49}$ Mingzhang Li, ${ }^{50}$ Yudong Qiu, ${ }^{51}$ Buqiang Wu, ${ }^{52}$ Jinfang Zheng, ${ }^{53}$ Xiaoliang Chen, ${ }^{54}$ Haihong Zhu, ${ }^{55}$ Kejun Hua, ${ }^{56}$ Maolin Yan, ${ }^{57}$ Peng Wang, ${ }^{58}$ Hong Zang, ${ }^{59}$ Xiaoming Ma, ${ }^{60}$ Jian Hong, ${ }^{61}$ Yingbin Liu (i) ${ }^{2,3}$
\end{abstract}

To cite: Ren T, Li Y, Zhang X, et al. Protocol for a gallbladder cancer registry study in China: the Chinese Research Group of Gallbladder Cancer (CRGGC) study. BMJ Open 2021;11:e038634. doi:10.1136/ bmjopen-2020-038634

- Prepublication history for this paper is available online. To view these files, please visit the journal online (http://dx.doi org/10.1136/bmjopen-2020038634).

Received 19 March 2020 Revised 11 November 2020 Accepted 29 November 2020

Check for updates

(c) Author(s) (or their employer(s)) 2021. Re-use permitted under CC BY-NC. No commercial re-use. See rights and permissions. Published by BMJ.

For numbered affiliations see end of article.

Correspondence to

Professor Yingbin Liu;

laoniulyb@shsmu.edu.cn

\section{ABSTRACT}

Introduction Gallbladder cancer (GBC), the sixth most common gastrointestinal tract cancer, poses a significant disease burden in China. However, no national representative data are available on the clinical characteristics, treatment and prognosis of GBC in the Chinese population.

Methods and analysis The Chinese Research Group of Gallbladder Cancer (CRGGC) study is a multicentre retrospective registry cohort study. Clinically diagnosed patient with GBC will be identified from 1 January 2008 to December, 2019 , by reviewing the electronic medical records from 76 tertiary and secondary hospitals across 28 provinces in China. Patients with pathological and radiological diagnoses of malignancy, including cancer in situ, from the gallbladder and cystic duct are eligible, according to the National Comprehensive Cancer Network 2019 guidelines. Patients will be excluded if GBC is the secondary diagnosis in the discharge summary. The demographic characteristics, medical history, physical examination results, surgery information, pathological data, laboratory examination results and radiology reports will be collected in a standardised case report form. By May 2021, approximately 6000 patient with GBC will be included. The clinical follow-up data will be updated until 5 years after the last admission for GBC of each patient. The study aimed (1) to depict the clinical characteristics, including demographics, pathology, treatment and prognosis of patient with $\mathrm{GBC}$ in China; (2) to evaluate the adherence to clinical guidelines of $\mathrm{GBC}$ and (3) to improve clinical practice for diagnosing and treating $\mathrm{GBC}$ and provide references for policy-makers. Ethics and dissemination The protocol of the CRGGC has been approved by the Committee for Ethics of Xinhua Hospital, Shanghai Jiao Tong University School of Medicine

\section{Strengths and limitations of this study}

- The Chinese Research Group of Gallbladder Cancer study is the first large-scale registry cohort study of gallbladder cancer (GBC) in China, covering 76 tertiary and secondary hospitals across 28 provinces.

- A standardised quality control and data management plan was designed to ensure the accuracy and reliability of the data.

- The electronic medical record systems are not consistent across hospitals, which may introduce variance in data recording and result in difficulty in systematic data formatting and integration.

- This is a retrospective study using convenience sampling. The study population may not be completely representative of patients with GBC in China.

- There is a lack of biospecimens from involved patients. The survival data are not validated through Chinese death registry.

(SHEC-C-2019-085). All results of this study will be published in peer-reviewed journals and presented at relevant conferences.

Trial registration number NCT04140552, Pre-results.

\section{INTRODUCTION}

Gallbladder cancer (GBC) is the most common type of biliary tract cancer ${ }^{1-3}$ and one of the most lethal malignancies, with a 5-year 
survival rate of $5 \%-15 \% .{ }^{145}$ Much effort has been made to optimise the treatment of GBC; however, the prognosis remains dismal ${ }^{4}{ }^{6}$, and the quality of current evidence for GBC is still far from perfect. Due to its relatively low incidence, clinical trials on GBC are difficult to conduct. Most recommendations and guidelines for GBC from the National Comprehensive Cancer Network (NCCN; 2019 V.4) and American Joint Committee on Cancer (AJCC; eighth version) were derived from evidence of moderate quality. ${ }^{78}$ Most of these studies were single-centre studies with limited sample sizes and generally no more than 300 cases,${ }^{9-11}$ which might introduce systematic bias into the conclusion.

On the other hand, common-used coding systems addressed little on GBC. The nomenclature of GBC in the literature is inconsistent. GBC defined by the AJCC eighth staging manual is a primary cancer in the gallbladder and cystic duct (C23.9 and part of C24.0; ICD-O-3 codes) ${ }^{8}$ However, many epidemiological studies refer to 'GBC' as 'GBC and extrahepatic cholangiocarcinoma (ECC; C23.9 and C24.0)', leading to confusion in its incidence, mortality and other epidemiological features. ${ }^{12} 13$ In addition, cystic duct cancer is undistinguishable from ECC in most cancer registry studies, which means that this specific subset of patients is likely to be omitted. ${ }^{14}$ Moreover, a commonly used coding system for surgery, the Facility Oncology Registry Data System, classifies GBC as 'all other sites', making it unlikely to define the extent of surgery and distinguish patients who undergo re-resection after GBC is incidentally found. ${ }^{15}$ Regarding regional lymph nodes, the Collaborative Stage (V.0204) system defines coeliac, superior mesenteric and para-aortic lymph nodes as regional nodes, which is not consistent with either the AJCC seventh or AJCC eighth definition. ${ }^{16}$ The coding problems in both patient identification and site-specific variables might lead to less stringent interpretation of the conclusions.

China is a high-GBC risk country, but little evidence has been based on the Chinese population. ${ }^{12}$ Data from GLOBOCAN show that, taking GBC and ECC together, the number of annual new cases in China accounts for $24.7 \%$ of new cases worldwide. ${ }^{13}$ Currently, the largest retrospective study of GBC in China was conducted by Zou and Zhang, ${ }^{17}$ including 3922 patients from 116 hospitals in 28 provinces of China during 1986-1998. This study described the demographic characteristics of GBC in China, without further data on detailed staging, treatment and prognosis information. Another study of 2379 patient with GBC from five northwestern provinces during $2009-2013^{18}$ reported that $55.1 \%$ of patient with GBC had advanced-stage tumours. Other reports were mainly single-centre studies with limited sample sizes. ${ }^{19} 20$ The critical characteristics in the diagnosis, treatment and prognosis of GBC in China are unknown.

Therefore, this study aimed to design a GBC cohort, the Chinese Research Group of Gallbladder Cancer (CRGGC) study, (1) to comprehensively evaluate the clinical characteristics, including demographics, pathology, treatment and prognosis of patient with GBC in China; (2) to evaluate adherence to clinical guidelines of GBC and (3) to improve clinical practice and guidelines for GBC and provide references for policy-makers.

\section{Methods and analysis}

Registry design

The CRGGC study is a multicentre retrospective registry cohort study. The project was launched by the Shanghai Key Laboratory of Biliary Tract Disease Research, with collaborators from 76 tertiary and secondary hospitals across 28 provinces in China (until 8 March 2020; see online supplemental file 1 ). We will review the electronic medical records (EMRs) of all diagnosed patient with GBC from 1 January 2008 to December 2019, and extract the related clinical and treatment information. The clinical follow-up data will be updated until 5 years after the last admission of each patient with GBC.

\section{Patient enrolment}

Patients will be identified with various search strategies: (1) ICD-10 code equals C23.9, or C24.0 with 'cystic duct'; (2) discharge diagnosis includes 'gallbladder cancer' (search strategy in Chinese: ((“胆囊”) AND (“ 癌” OR “恶性肿瘤” OR “占位”), which means “gallbladder" /"cystic duct" AND ("cancer" OR "malignancy" OR "space-occupying lesion")); and (3) pathological reports include "gallbladder cancer". All three search strategies will be applied in each centre. The results will be merged for subsequent exclusion. These search strategies are designed to be redundant because some search strategies may not be applicable in specific EMR systems and in specific periods.

All identified admissions to the hospital will be manually filtered according to the diagnostic criteria of the NCCN 2019 V.4 guidelines for hepatobiliary cancer. ${ }^{7}$ Patients with a pathological or radiological diagnosis of malignancy, including cancer in situ, from the gallbladder and cystic duct are eligible. Patients will be excluded if GBC is the secondary diagnosis in the discharge summary because patients admitted for other diseases are likely to have obscure cancer traits.

The study will include patients diagnosed before December 2019. According to our preliminary estimation, more than 6000 cases will meet our inclusion criteria. We expect to finish data collection by May 2021. After finishing enrolment of a short-term target of 2000 cases, a primary analysis will be performed. The follow-up will be updated until 5 years after the admission of each patient. More centres are expected to participate in the CRGGC study; thus, the collaborator list may be expanded.

\section{Clinical outcomes and follow-up}

The main outcome is the 5-year overall survival (OS). OS is defined as the duration from the date of first diagnosis to the date of death, and it is censored at the date of the last follow-up when the patients are alive. We will also include the following outcomes: progression-free survival 


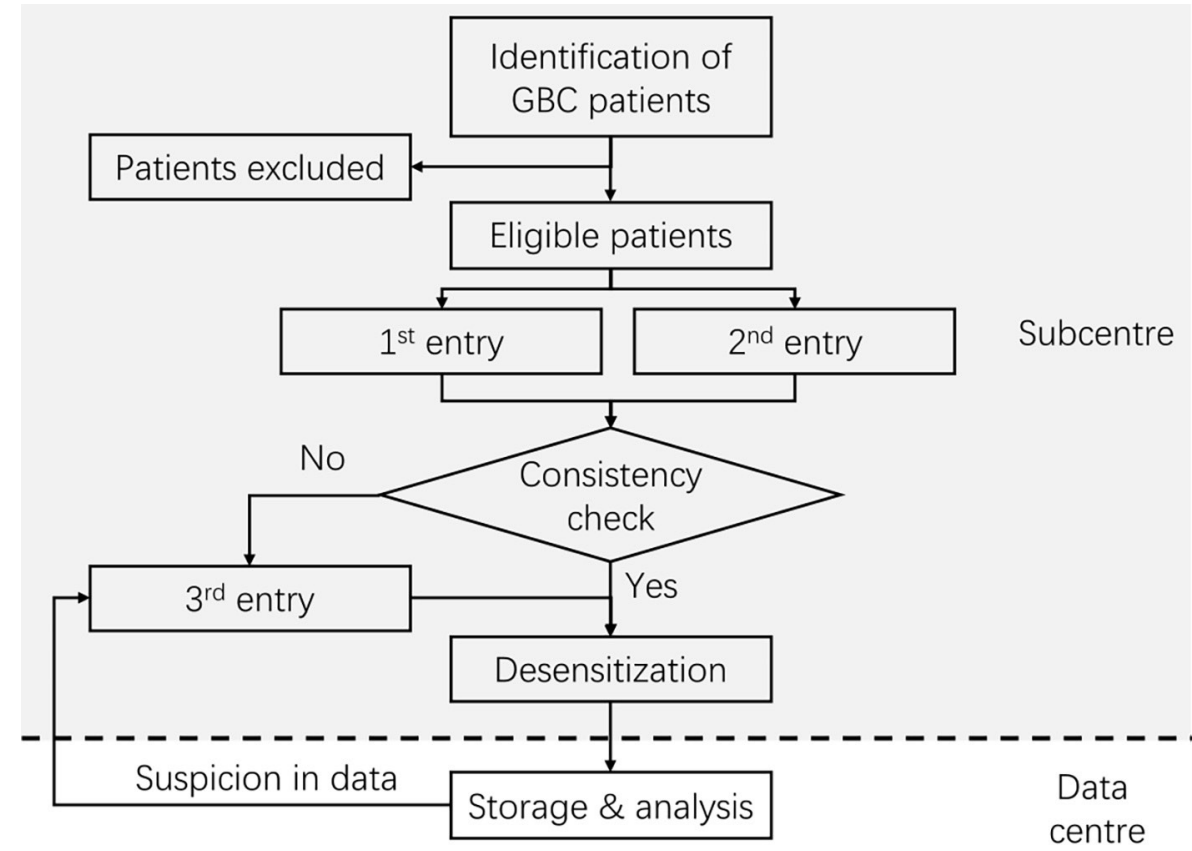

Figure 1 Workflow of data collection and quality control in CRGGC. CRGGC, Chinese Research Group of Gallbladder Cancer; GBC, gallbladder cancer.

(PFS), defined as the duration between the date of first diagnosis and the date of recurrence, and censored at the date of the last follow-up when the patients have no evidence of recurrence; cancer-specific survival defined as the duration between the date of first diagnosis and the date of cancer-caused death, and censored at the date of the last follow-up when the patients are alive or died from other causes; 3-year OS; and 90-day mortality (for patients who undergo surgery), which will be used to indicate perioperative mortality. Clinical follow-up is defined as the routine practice of hospitals of collecting patient data on treatment, tumour recurrence and patient survival, either by outpatient/inpatient records or telephone. We require hospitals to equip such a system and at least one follow-up per year to join our collaboration. Based on these data, we will update patients' follow-up statuses every 12 months. The data being collected from clinical follow-up will include date of recurrence, date of death, date of last contact, whether reresection is performed if the malignancy is found incidentally, and whether the patient receives adjuvant therapy.

\section{Data collection}

The workflow of data collection and quality control is shown in figure 1 . Before data collection, a group of hepatobiliary specialists designed a structured case report form, aiming to delineate features of patients with GBC and answer corresponding clinical questions. The case report form includes the following information: demographic characteristics, medical history, physical examination results, surgery information, pathological data, laboratory examination results and radiology reports. We have compiled a codebook to standardise the definition of each variable. The data centre will be responsible for training doctors to collect data. Data collection will be carried out by using EpiData (V.4.6.0.2, EpiData Association, Denmark).

Automated logic checks will be applied to prevent outof-range values. Duplicated entry will be required. If any discrepancies are found, a third specialist will be brought in for discussion and make a final decision.

After data entry and quality control in each centre, the data will be anonymised and transferred to the servers in the data centre. The data centre is located at Shanghai Key Laboratory of Biliary Tract Disease Research, which is equipped with data servers and essential firewall and backup systems. The data centre will be responsible for quality assessment, storage, sharing and analysis of the data. A group of researchers in the data centre will manage the database.

The data manager will assess the quality of the data after transfer to the data centre. The assessment is based on the structure of missing data and a comparison to baseline data. First, we will apply a grading system, where variables are classified into essential, important and normal importance. Based on the proportion of missing values in each category, the entries will be graded as level A, B, C or $\mathrm{D}$ in quality. Entries of category D quality will be normally excluded from analysis. Second, outliers and inconsistent data will be identified. Third, we will compare baseline characteristics of the new data to previous data, with indicators including sex ratio, mean age, proportion of tumor, node, and metastasis (TNM) stage, and 5-year OS. We will apply $\chi^{2}$ test, t-test and log-rank test between the two datasets. When a significant difference is found, the data manager will analyse and record suspicious data. The data manager will inquire about the data in question 
with the data source and ask for confirmation. The desensitised data will be accessible to collaborators after the completion of the database. A research proposal to the CRGGC Scientific Committee will be essential for analysis of the data.

\section{Demographic data and medical history}

The EMR data for each patient will be collected for every hospital visit from 1 January 2008. The baseline data will be retrieved, including the following aspects: (1) demographics: age at diagnosis, sex, race and date of diagnosis; (2) medical history: emergency operation, chief complaint, endoscopic retrograde cannulation of the pancreas (ERCP) performed within 30 days before surgery, percutaneous transhepatic cholangial drainage (PTCD) performed within 30 days before surgery, neoadjuvant therapy and method of diagnosis (pathology, radiology or other); (3) medical history: history of gallstone, history of gallbladder polyps, history of other malignancies, hypertension, diabetes mellitus and other comorbidities; (4) social and personal history: marital status, smoking history and use of alcohol; and (5) other aspects: weight, height, family history and total expenditure.

\section{Surgery information}

1. The preoperative and intraoperative diagnoses will be recorded. A diagnosis of 'GBC', 'gallbladder tumour', or 'space-occupying lesion in gallbladder' is regarded as the detection of malignancy.

2. Regional lymphadenectomy requires the resection of hilar nodes. ${ }^{8}$ Further clearance of lymph nodes is classified as extended lymphadenectomy.

3. The extent of lymphadenectomy includes the cystic duct, common bile duct, portal vein, hepatic artery, common hepatic artery, postsuperior pancreatic, coeliac, superior mesenteric, suprapyloric, left gastric artery and paraaortic lymph nodes. ${ }^{821} 22$

4. Combined hepatectomy is classified as no hepatectomy, liver wedge resection/partial hepatectomy, $\mathrm{IVb}+\mathrm{V}$ segmentectomy, hemihepatectomy, extent more than hemihepatectomy, radiofrequency ablation and hepatectomy for other reasons.

5. If the malignancy is diagnosed after surgery, further treatment information may not be available (the patient may turn to a second hospital for reresection). Patients in this case will be categorised as 'simple cholecystectomy performed; further treatment not available'. If reresection is available, its operative reports will be reviewed as previously mentioned.

6. ERCP, PTCD and transarterial chemoembolisation are not defined as surgery but as supportive treatment.

7. Palliative surgery is defined as resection of the primary tumour, reconstruction of the digestive tract, or both when there is evidence of distant metastasis or unresectable tumour.

Other surgery-related variables include date of surgery, laparoscopic surgery, combined bile duct resection, tumour positioned on the hepatic or peritoneal side, perivascular invasion, perforation, porcelain gallbladder, duration of surgery, intraoperative blood loss and American Society of Anesthesiologists score.

\section{Pathological data}

Pathological data will be recorded, including size of the tumour (in three dimensions), resection margin, tumour positioned on the hepatic or peritoneal side, tumour positioned on the fundus, body, neck or cystic duct, depth of invasion (carcinoma in situ or lamina propria, muscularis, perimuscular connective tissue, full layer, serosa, adjacent organ or major vascular invasion $^{8}$ ), liver invasion, number of nodes examined, positive lymph nodes, number of hilar nodes examined, positive hilar lymph nodes, region of positive nodes, region of nodes examined (with codes the same as those used for the region of lymphadenectomy in surgery), grade, histology type (using ICD-O-3 codes), ${ }^{23}$ microvascular invasion and perineural invasion. Tumours will be staged according to the AJCC eighth staging manual according to pathological reports derived from the aforementioned variables. Notably, the description of 'invasion of full layer' for depth of invasion is not suggested in the AJCC eighth manual but is commonly used in China.

\section{Laboratory examination}

Laboratory examination results for patients will be collected with the date of examination. Indicators of interest include the following: (1) routine blood tests: white cell count, haemoglobin and platelet count; (2) liver function tests: total bilirubin, direct bilirubin, albumin, alanine aminotransferase, aspartate aminotransferase, alkaline phosphatase and gamma-glutamyl transferase; (3) renal function tests: blood urea nitrogen and creatine; (4) lipid indicators: triglycerides and total cholesterol; (5) inflammation indicators: $\mathrm{C}$ reactive protein and lactic dehydrogenase; (6) coagulation indicators: international normalised ratio, prothrombin time, activated partial thromboplastin time and fibrinogen; (7) tumour markers: carcinoembryonic antigen, carbohydrate antigen 19-9, carbohydrate antigen 125 and alpha fetoprotein; and (8) other tests: blood type and hepatitis $\mathrm{B}$ test. The test method and normal range of each indicator may vary across hospitals. Thus, we will first uniform the units of each indicator according to the first enrolled hospital; then, based on the first enrolled hospital, we will normalise each result of laboratory examination by its normal range across different hospitals.

\section{Radiology reports}

Radiological reports will be collected with the date of examination. The following indicators will be collected: the type of examination (ultrasound, CT, MRI and/ or other types) and the conclusion of the examination (inflammation, polyp, tumour, gallstone and/or others).

\section{Statistical analysis}

The continuous variables will be described visually by histogram and summarised as mean $\pm \mathrm{SD}$ or median 
(IQR), for normal-distributed and skewed-distributed values. The discrete variables will be summarised as frequency (percentage). The proportion of missing data will be described, and an indicator of missing will be deployed for analysis. Differences with a two-sided $\mathrm{p}<0.05$ are considered as statistically significant.

We will calculate the average number of GBC diagnosed per year in each hospital, because the volume for patients with cancer showed impact on patients' characteristics, treatment modalities and prognosis. ${ }^{24}$ The distribution of hospitals' average patients with GBC per year will be described in bar plot; 1-2 cut-off points will be determined by inspecting the pattern to classify hospitals into low volume and high volume. Correlation between hospital volume and patients' characteristics will be shown by either Pearson's R, Spearman's r, or $\chi^{2}$, whichever appropriate. Potential correlated variables includes (but not limited to) sex, age at diagnosis, TNM stage, gallstone, surgery type and adjuvant therapy.

Time trends for age, sex, TNM stage, surgery type, adjuvant therapy and diagnosis time will be shown by scatter plot fitted by linear or locally estimated scatterplot smoothing, whichever appropriate.

The median survival time and lost to follow-up rate will be described. The Kaplan-Meier method will be applied to assess the survival of patients, stratified by TNM stage and by whether surgery performed. Cox proportional hazards regression will be applied to evaluate predictors of prognosis, including (but not limited to) age, sex, T stage, $\mathrm{N}$ stage, $\mathrm{M}$ stage, adjuvant therapy, extent of resection, gallstone, resection margin, histological grade, perineural invasion and microvascular invasion.

As proposed in both NCCN and Chinese Medical Association guidelines, ${ }^{7}{ }^{25}$ GBC more advance than T1b requires resection of liver bed and regional lymphadenectomy. Moreover, patients with nodal metastasis are recommended to take chemotherapy. These three indicators will be described to evaluate the adherence to clinical guidelines of GBC. Also, their significance in prognosis will be tested by survival analysis.

\section{Patient and public involvement}

Patients or the public involvement are not in the plans of this research.

\section{Ethics and dissemination}

The protocol of the CRGGC has been approved by the Committee for Ethics of Xinhua Hospital, Shanghai Jiao Tong University School of Medicine (SHEC-C-2019-085). All results of this study will be published in peer-reviewed journals and presented at relevant conferences.

\section{DISCUSSION}

The CRGGC study is a large multicentre registry cohort study to evaluate the clinical presentation, treatment and prognosis of patients with GBC in China. The focus of CRGGC is to (1) describe the status quo of treatment and survival of patient with GBC in China and (2) improve the standardised treatment of GBC.

On reviewing the published studies on GBC, we found a lack of large observational studies on GBC in China focusing on its clinical features and prognosis. Moreover, international studies on GBC were limited by small sample sizes and inconsistent coding systems for GBC. Our data will establish a collaborative platform for GBC research, providing valuable data from China.

GBC is a relatively rare but lethal malignancy, making it important to address the standardisation of its primary care, treatment and post-treatment follow-up. Researchers have shown unsatisfactory adherence to clinical guidelines. Radical cholecystectomy was recommended for T1b GBC by the NCCN guidelines nearly 10 years ago; however, only $50 \%$ of T1b patientswith GBC in the USA received radical cholecystectomy. ${ }^{14}$ Bergquist et $a l^{26}$ reported that from 2004 to 2012 , only $28.2 \%$ of patients with GBC with positive nodal disease received adjuvant chemotherapy in the National Cancer Database of the USA even though this was the recommended treatment in the NCCN guidelines. Knowing the current situation is essential for future improvement; however, no data have been reported on GBC treatment in China. Well-designed observational studies in China will help to point out weakness in clinical practice and, at the same time, summarise valuable clinical experience in the treatment of $\mathrm{GBC}$ and pave the way for further standardised treatment.

GBC cases in China account for nearly $1 / 4$ of cases worldwide; thus, GBC poses a significant disease burden in China. However, few clinical studies of the diagnosis and treatment of GBC have been performed in China, making this significant population under-represented. By launching the CRGGC study, we also expect to boost collaborations among Chinese researchers. We hope this collaboration could induce further translational research and clinical trials in China, providing essential evidence on GBC treatment.

There are several limitations and potential biases in our study design. (1) The retrospective nature is inevitably related to information bias and heterogeneity in the data recording. This will cause difficulty in the standardisation of data and a relatively large proportion of missing data. To overcome such bias, we composed and continue to update a codebook for standardisation of each variable. Researchers responsible for data entry are trained and qualified at the data centre. The missing data are analysed to determine potential bias. (2) This is a retrospective study using convenience sampling. Thus, the cohort may not be completely representative of patient with GBC in China. However, we attempt to include centres in every province in China. Moreover, most patients with cancer in China are treated in tertiary hospitals. (3) Biospecimens of the involved patients are not collected. Future collaboration on this issue will be considered. (4) As patients with incidental GBC may turn to other hospitals for reresection, resulting in incomplete treatment information. We addressed this problem by defining these patients separately to aid further sensitivity analysis. (5) Currently, we have not made collaboration with Chinese death registry, thus, part of the death information may be lost and the follow-up 
data might be biased due to lack of validation. On the one hand, the CRGGC study actively seek cooperation with relevant registries; on the other hand, we require collaborated hospitals to equip clinical follow-up system, compare prognosis data in each hospital to identify systematic bias, and update follow-up data yearly.

\section{Author affiliations}

${ }^{1}$ Department of General Surgery, Xinhua Hospital Affiliated to Shanghai Jiaotong University School of Medicine, Shanghai, China

${ }^{2}$ Shanghai Key Laboratory of Biliary Tract Disease Research, Shanghai, China

${ }^{3}$ Department of Biliary-Pancreatic Surgery, Shanghai Jiao Tong University School of Medicine Affiliated Renji Hospital, Shanghai, Shanghai, China

${ }^{4}$ Clinical Research Unit, Xinhua Hospital Affiliated to Shanghai Jiaotong University

School of Medicine, Shanghai, China

${ }^{5}$ Department of General Surgery, Shanxi Provincial Cancer Hospital, Taiyuan, Shanxi, China

${ }^{6}$ Department of Hepatobiliary Surgery, The First Affiliated Hospital of Xi'an Jiaotong University, Xi'an, Shaanxi, China

${ }^{7}$ Department of Hepatobiliary Surgery, People's Hospital of Hunan Province, Changsha, Hunan, China

${ }^{8}$ Department of HPB Surgery, The First Affiliated Hospital of Harbin Medical University, Harbin, Heilongjiang, China

${ }^{9}$ Department of Hepatobiliary Surgery, Shandong Provincial Hospital, Jinan, Shandong, China

${ }^{10}$ Department of Hepatobiliary Surgery, Xijing Hospital, Xi'an, Shaanxi, China

${ }^{11}$ Department of HPB Surgery, Sir Run Run Shaw Hospital, Zhejiang University

School of Medicine, Hangzhou, Zhejiang, China

${ }^{12}$ Department of Pancreatobiliary Surgery, Tongji Hospital, Tongji Medical College of Huazhong University of Science and Technology, Wuhan, Hubei, China

${ }^{13}$ Department of Biliary Surgery, Eastern Hepatobiliary Surgery Hospital, Shanghai, China

${ }^{14}$ Department of HPB Surgery, The Second Hospital of Jilin University, Changchun, Jilin, China

${ }^{15}$ Department of General Surgery, Shanghai General Hospital, Shanghai, China

${ }^{16}$ Department of Surgical Oncology, The First Affiliated Hospital of Bengbu Medical College, Bengbu, Anhui, China

${ }^{17}$ Department of General Surgery, The First Affiliated Hospital of Wenzhou Medical University, Wenzhou, Zhejiang, China

${ }^{18}$ Department of HPB Surgery, Harbin Medical University Cancer Hospital, Harbin, Heilongjiang, China

${ }^{19}$ Department of Hepatobiliary Surgery, Shengjing Hospital of China Medical

University, Shenyang, Liaoning, China

${ }^{20}$ Department of HPB Surgery, The Affiliated Hospital of Qingdao University, Qingdao, Shandong, China

${ }^{21}$ Department of Gl Surgery, China-Japan Union Hospital of Jilin University, Changchun, Jilin, China

${ }^{22}$ Department of Gl Surgery, Shaoxing People's Hospital, Shaoxing, Zhejiang, China

${ }^{23}$ Department of General Surgery, Shaoxing Second Hospital, Shaoxing, Zhejiang, China

${ }^{24}$ Department of GI Surgery, The First People's Hospital of Taicang, Taicang, Jiangsu, China

${ }^{25}$ Department of Hepatobiliary Surgery, Wuxi Second People's Hospital, Wuxi,

Jiangsu, China

${ }^{26}$ Department of Hepatobiliary Surgery, Nantong Tumor Hospital, Nantong, Jiangsu, China

${ }^{27}$ Department of General Surgery, Shanghai Jiaotong University School of Medicine Xinhua Hospital Chongming Branch, Shanghai, China

${ }^{28}$ Department of General Surgery, Changshu No. 1 People's Hospital, Changshu, Jiangsu, China

${ }^{29}$ Department of Pancreatic Surgery, The First People's Hospital of Taicang, Urumqi, Xinjiang, China

${ }^{30}$ Department of General Surgery, Tongliao City Hospital, Inner Mongolia, Tongliao, China

${ }^{31}$ Department of Hepatobiliary Surgery, The Second Affiliated Hospital of Nanchang University, Nanchang, Jiangxi, China

${ }^{32}$ Department of General Surgery, The First Affiliated Hospital of Nanchang University, Nanchang, Jiangxi, China
${ }^{33}$ Department of HPB Surgery, Changzhou No. 2 People's Hospital, Changzhou, Jiangsu, China

${ }^{34}$ Department of General Surgery, The First Affiliated Hospital of Kunming Medical

University, Kunming, Yunnan, China

${ }^{35}$ Department of General Surgery, Shanghai Fengxian District Central Hospital,

Shanghai, Shanghai, China

${ }^{36}$ Department of HPB Surgery, The Fifth Affiliated Hospital of Zhengzhou University, Zhengzhou, Henan, China

${ }^{37}$ Department of General Surgery, Taizhou Municipal Hospital, Taizhou, Zhejiang, China

${ }^{38}$ Department of HPB Surgery, The First Affiliated Hospital of Anhui Medical

University, Hefei, Anhui, China

${ }^{39}$ Department of Pancreatobiliary Surgery, The Second Affiliated Hospital of Harbin Medical University, Harbin, Heilongjiang, China

${ }^{40}$ Department of Gl Surgery, Xuzhou Central Hospital, Xuzhou, Jiangsu, China

${ }^{41}$ Department of HPB Surgery, Xinghua City People's Hospital, Taizhou, Jiangsu,

China

${ }^{42}$ Department of General Surgery, Zhongshan Hospital, Fudan University, Shanghai, China

${ }^{43}$ Department of General Surgery, Henan Cancer Hospital, Zhengzhou, Henan, China ${ }^{44}$ Department of Pancreatobiliary Surgery, Sun Yat-Sen Memorial Hospital, Sun YatSen University, Guangzhou, Guangdong, China

${ }^{45}$ Department of Pancreatic Surgery, Tianjin Medical University Cancer Hospital,

Tianjin, China

${ }^{46}$ Department of HPB Surgery, The First Bethune Hospital of Jilin University,

Changchun, Jilin, China

${ }^{47}$ Department of Abdominal Surgery, Jiangxi Cancer Hospital, Nanchang, Jiangxi, China

${ }^{48}$ Department of General Surgery, The First Hospital of Lanzhou University, Lanzhou, Gansu, China

${ }^{49}$ Department of General Surgery, First Hospital of Shanxi Medical University, Taiyuan, Shanxi, China

${ }^{50}$ Department of General Surgery, Baotou City Central Hospital, Baotou, Inner Mongolia, China

${ }^{51}$ Department of Pancreatobiliary Surgery, Nanjing Drum Tower Hospital, the Affiliated Hospital of Nanjing University Medical School, Nanjing, Jiangsu, China ${ }^{52}$ Department of General Surgery, Heping Hospital Affiliated to Changzhi Medical College, Changzhi, Shanxi, China

${ }^{53}$ Department of General Surgery, Hainan Provincial People's Hospital, Haikou, Hainan, China

${ }^{54}$ Department of Hepatobiliary Surgery, Jiangxi Provincial People's Hospital, Nanchang, Jiangxi, China

${ }^{55}$ Department of General Surgery, Qinghai Provincial People's Hospital, Xining, Qinghai, China

${ }^{56}$ Department of General Surgery, Yinzhou Hospital Affiliated to Medical School of Ningbo University, Ningbo, Zhejiang, China

${ }^{57}$ Department of Hepatobiliary Surgery, Fujian Provincial Hospital, Fuzhou, Fujian, China

${ }^{58}$ Department of HPB Surgery, Affiliated Hospital of Nantong University, Nantong, Jiangsu, China

${ }^{59}$ Department of Hepatobiliary Surgery, The First People's Hospital of Nantong, Nantong, Jiangsu, China

${ }^{60}$ Department of General Surgery, The Second Affiliated Hospital of Suzhou University, Suzhou, Jiangsu, China

${ }^{61}$ Department of General Surgery, The Affiliated Hospital of Xuzhou Medical University, Xuzhou, Jiangsu, China

Acknowledgements We would like to thank Professor Wenyi Yang at Shanghai General Hospital, China, and Professor Jiong Li at Aarhus University, Denmark, for their valuable advice on the CRGGC registry. We thank our collaborators for their contributions to the CRGGC study, as follows: Professor Lianxin Liu at Anhui Provincial Hospital, Professor Xu Liu at Peking University Shenzhen Hospital, Professor Yinmo Yang at Peking University First Hospital, Professor Qiang Xu at Peking Union Medical College Hospital, Professor Banghao Xu at The First Affiliated Hospital of Guangxi Medical University, Professor Jianhua Liu at The Second Hospital of Hebei Medical University, Professor Baobiing Yin at Huashan Hospital, Professor Weilong Cai at Huzhou Central Hospital, Professor Zhiping Zhang at Ningbo First Hospital, Professor Xuting Zhi at Qilu Hospital of Shandong University, Professor Longhua Rao at Central Hospital of Minhang District, Professor Xiaoping 
Yang at Shanghai Pudong Hospital, Professor Jiahua Yang at Putuo District People's Hospital of Shanghai, Professor Ruiwu Dai at Chengdu Military General Hospital, Professor Leida Zhang at Southwest Hospital, Professor Xinbao Wang at Zhejiang Cancer Hospital, Professor Jinhui Zhou at The Second Affiliated Hospital of Zhejiang University School of Medicine, Professor Yongjun Chen at Ruijin Hospital, and Professor Yongwei Sun at Renji Hospital. We would also like to thank the researchers who made significant contributions to the CRGGC registry, as follows: Professor Min Wang at Tongji Hospital, Professor Rongsheng Zhang at Shanxi Provincial Cancer Hospital, Dr Kai Qu at The First Affiliated Hospital of Xi'an Jiaotong University, Professor Lei Zou at The First Affiliated Hospital of Kunming Medical University, Professor Fubao Liu at The First Affiliated Hospital of Anhui Medical University, Professor Leibo Xu at Sun Yat-Sen Memorial Hospital, Sun Yat-Sen University, Professor Xianhai Mao at People's Hospital of Hunan Province, Professor Ling Zhang at Henan Cancer Hospital, Dr Zhizhen Li at Eastern Hepatobiliary Surgery Hospital, and Dr Lei Wang at Wuxi Second People's Hospital.

Contributors $\mathrm{YL}$ is the principal investigator steering the CRGGC, and responsible for conceptualisation, funding acquisition and supervision of the study. TR, YL, XZ and YG wrote and revised the manuscript.TR, YL,YG,ZS, ML, XW, X-AW, WW, YS, $R B, W G$ and $P D$ discussed and drafted the case report form, standard operation procedure in data collection and management, and standard of quality control. TR and $Y L$ are responsible for data curation and coordination.XZ is responsible for the methodology. TR, $Y L$ and $X Z$ will be responsible for data analysis and reporting of the work. XD, ChanL, ChangL, BS, JL, LW, DH, RQ, XJ,XZ, JX, JJ, BY, BL, CD, JC, HC, FT,ZZ, YW, HJ, HC, ZF, JG, WH, XF, LF, LZ, CZ, KW, XZ, XL, CJ, YQ, YC, YX, XW, HL, YH, $\mathrm{CL}, \mathrm{JH}, \mathrm{CW}, \mathrm{QL}, \mathrm{XL}, \mathrm{JL}, \mathrm{ML}, \mathrm{YQ}, \mathrm{BW}, \mathrm{JZ}, \mathrm{XC}, \mathrm{HZ}, \mathrm{KH}, \mathrm{MY}, \mathrm{PW}, \mathrm{HZ}, \mathrm{XM}, \mathrm{JH}, \mathrm{WG}$ and $\mathrm{YL}$ are responsible for resources, data collection and quality control in collaborated hospitals. All authors reviewed the manuscript for intellectual content and approved the final version of the report.

Funding This study was supported by the National Natural Science Foundation of China (No. 31620103910,81874181, and 91940305), the Emerging Frontier Programme of Hospital Development Centre (No. SHDC12018107), the Key Programme of Shanghai Science and Technology Commission (No. YDZX20193100004049), the Shanghai Key Laboratory of Biliary Tract Disease Research Foundation (17DZ2260200), the Shanghai Artificial Intelligence Innovation and Development Project (2019, Project of Xinhua Hospital Affiliated to Shanghai Jiao Tong University School of Medicine), the Multi-Centre Clinical Research Project of Shanghai Jiao Tong University School of Medicine (DLY201507) and the Translational Medicine Innovation Fund of Shanghai Jiao Tong University School of Medicine (15ZH4003).

Competing interests None declared.

Patient consent for publication Not required.

Provenance and peer review Not commissioned; externally peer reviewed.

Supplemental material This content has been supplied by the author(s). It has not been vetted by BMJ Publishing Group Limited (BMJ) and may not have been peer-reviewed. Any opinions or recommendations discussed are solely those of the author(s) and are not endorsed by BMJ. BMJ disclaims all liability and responsibility arising from any reliance placed on the content. Where the content includes any translated material, BMJ does not warrant the accuracy and reliability of the translations (including but not limited to local regulations, clinical guidelines, terminology, drug names and drug dosages), and is not responsible for any error and/or omissions arising from translation and adaptation or otherwise.

Open access This is an open access article distributed in accordance with the Creative Commons Attribution Non Commercial (CC BY-NC 4.0) license, which permits others to distribute, remix, adapt, build upon this work non-commercially, and license their derivative works on different terms, provided the original work is properly cited, appropriate credit is given, any changes made indicated, and the use is non-commercial. See: http://creativecommons.org/licenses/by-nc/4.0/.

\section{ORCID iDs}

Tai Ren http://orcid.org/0000-0001-5864-9425

Xi Zhang http://orcid.org/0000-0002-4524-2084

Renyi Qin http://orcid.org/0000-0001-5079-5137

Yingbin Liu http://orcid.org/0000-0001-6110-0185
REFERENCES

1 Hundal R, Shaffer EA. Gallbladder cancer: epidemiology and outcome. Clin Epidemiol 2014;6:99-109.

2 Misra S, Chaturvedi A, Misra NC, et al. Carcinoma of the gallbladder. Lancet Oncol 2003;4:167-76.

3 Li M, Zhang Z, Li X, et al. Whole-Exome and targeted gene sequencing of gallbladder carcinoma identifies recurrent mutations in the ErbB pathway. Nat Genet 2014;46:872-6.

4 Lindnér P, Holmberg E, Hafström L. Gallbladder cancer - no improvement in survival over time in a Swedish population. Acta Oncol 2018;57:1482-9.

5 Surveillance, epidemiology, and end results (SEER) program (www. seer.cancer.gov) research data (1973-2016), National cancer Institute, DCCPS, surveillance research program, released April 2019, based on the may 2019 submission.

6 Creasy JM, Goldman DA, Gonen M, et al. Evolution of surgical management of gallbladder carcinoma and impact on outcome: results from two decades at a single-institution. HPB 2019;21:1541-51.

7 NCCN clinical practice guidelines in oncology: hepatobiliary cancers, version 4. 2019: national comprehensive cancer network, 2019. Available: https://www.nccn.org/professionals/physician_gls/pdf/ hepatobiliary.pdf [Accessed 2020-02-13]

8 American Joint Committee on Cancer. Ajcc cancer staging manual. 8th Edition, 2017.

9 Shindoh J, de Aretxabala X, Aloia TA, et al. Tumor location is a strong predictor of tumor progression and survival in T2 gallbladder cancer: an international multicenter study. Ann Surg 2015;261:733-9.

10 Amini N, Kim Y, Wilson A, et al. Prognostic implications of lymph node status for patients with gallbladder cancer: a multi-institutional study. Ann Surg Oncol 2016;23:3016-23.

11 Ito $\mathrm{H}$, Ito K, D'Angelica M, et al. Accurate staging for gallbladder cancer: implications for surgical therapy and pathological assessment. Ann Surg 2011;254:320-5.

12 Tuo J, Zhang M, Zheng R. [Report of incidence and mortality of gallbladder cancer in China, 2014]. Chin J Oncol 2018;40:894-9.

13 Bray F, Ferlay J, Soerjomataram I, et al. Global cancer statistics 2018: GLOBOCAN estimates of incidence and mortality worldwide for 36 cancers in 185 countries. CA Cancer J Clin 2018;68:394-424.

14 Vo E, Curley SA, Chai CY, et al. National failure of surgical staging for T1b gallbladder cancer. Ann Surg Oncol 2019;26:604-10.

15 American College of Surgeons. Facility oncology registry data system. 3rd ed. Chicago, IL, 2002.

16 Collaborative Stage Work Group of the American Joint Committee on Cancer. Collaborative stage data collection system user documentation and coding instructions. version 02.04. Chicago, IL: American Joint Committee on Cancer, 2012.

17 Zou S, Zhang L. [Clinical epidemiologic characteristics of carcinoma of gallbladder in China]. Chinese Journal of Practical Surgery 2000;20:43-6.

18 Shen $\mathrm{H}$, Song $\mathrm{H}$, Wang L. [Clinical feature of gallbladder cancer in Northwestern China: a report of 2379 cases from 17 institutions]. Chinese Journal of Surgery 2015;53:747-51.

19 Hsing AW, Gao YT, Devesa SS, et al. Rising incidence of biliary tract cancers in Shanghai, China. Int J Cancer 1998;75:368-70.

20 Bao P-P, Zheng Y, Wu C-X, et al. Cancer incidence in urban Shanghai, 1973-2010: an updated trend and age-period-cohort effects. BMC Cancer 2016;16:284.

21 Brierley JD, Gospodarowicz MK, Wittekind C. Tnm classification of malignant tumours. 8th ed. Wiley, 2018.

22 Kishi Y, Shimada K, Hata S, et al. Definition of T3/4 and regional lymph nodes in gallbladder cancer: which is more valid, the UICC or the Japanese staging system? Ann Surg Oncol 2012;19:3567-73.

23 World Health Organization. International classification of diseases for oncology (ICD-O) - 3rd edition, 1st revision. 3rd ed.. ed. Geneva, 2013.

24 Hata T, Motoi F, Ishida M, et al. Effect of hospital volume on surgical outcomes after pancreaticoduodenectomy: a systematic review and meta-analysis. Ann Surg 2016;263:664-72.

25 Chinese Society of Biliary Surgery. [Guidelines for the diagnosis and treatment of gallbladder carcinoma (2015 edition)]. Chin J Dig Surg 2015;14:881-90.

26 Bergquist JR, Shah HN, Habermann EB, et al. Adjuvant systemic therapy after resection of node positive gallbladder cancer: time for a well-designed trial? (results of a US-national retrospective cohort study). Int J Surg 2018;52:171-9. 